Volume 8, No.6, November - December 2019

International Journal of Advanced Trends in Computer Science and Engineering

Available Online at http://www.warse.org/LJATCSE/static/pdf/file/ijatcse154862019.pdf

https://doi.org/10.30534/ijatcse/2019/154862019

\title{
Challenge of 5G Network Technology for Telemedicine and Telesurgery
}

\author{
${ }^{1}$ Josua Setyo, ${ }^{2}$ Alvin Gunawan. ${ }^{3}$ Muhammad Rizki Sani, Gunawan Wang ${ }^{4}$ \\ 1,2,3, Information Systems Management Department, BINUS Graduate Program-Master of Information Systems Management, \\ Bina Nusantara University, Jakarta, Indonesia. 11480. \\ E-mail: ${ }^{1}$ josua.setyo@binus.ac.id; ${ }^{2}$ alvin.setyo@binus.ac.id; ${ }^{3}$ muhammad.sani@binus.ac.id; ${ }^{4}$ gwang@ binus.edu.
}

\begin{abstract}
Telesurgery is one of the techniques for performing remote surgery with the use of robots that are controlled by surgeons in real time. as we know, that doing telesurgery requires network speed with near zero latency. With the latest communication technology called 5 Generation (5G), network speed is no longer an obstacle. But this can be done in countries that have adopted the $5 \mathrm{G}$ technology and there is still a lot of preparation needed by other countries to be able to maximize this $5 \mathrm{G}$ technology especially in the field of telemedicine. In this study, we will explain what is the challenge applying telesurgery with the help of 5G connections, especially in hospitals that have limited network connections.
\end{abstract}

Key words : Telesurgery, Telemedicine, 5 Generation, Networking

\section{INTRODUCTION}

Over the past few years, communication technology especially in the network, has continued to innovate with purpose to increase bandwidth and lower latency. This is done from the 1st generation to the 4th generation or called Long Term Evolution-Advanced (LTE-A)[1]. This year, network and communication technology has entered the 5th generation $(5 \mathrm{G})$ where the 5 th generation is believed to be able to change the existing business strategies in the world. Following are the specifications offered when $5 \mathrm{G}$ has been implemented globally in Figure 1.

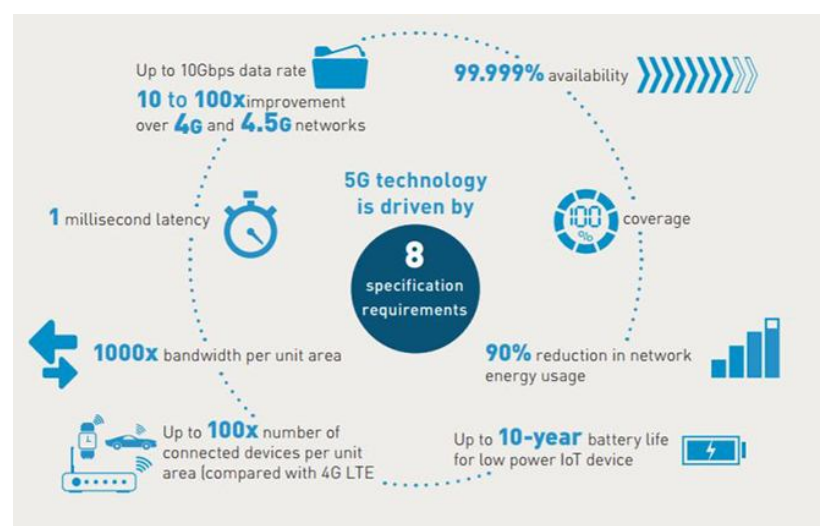

Figure 1 : Specifications offered by $5 \mathrm{G}$
With the various advantages offered by $5 \mathrm{G}$ compared to previous generations, it allows several new methods in business processes such as controlling drones, unmanned cars, smart cities, and of course in the medic field. In this essay, it will be more emphasized on the use of $5 \mathrm{G}$ networks to help medical treatment or remote operation in real time.

\section{Application of Telemedicine and Telesurgery}

Telemedicine is a blend of knowledge between communication technology and also medical use for remote medical services. This technology utilizes satellites to broadcast consultations to health facilities using Video Conference media. The history of telemedicine has existed from the days of World War 2 where doctors used telegram to help instruct war soldiers in handling emergency medicine. As communication technology developed, telemedicine became very reliable in utilizing media such as radio, television, telephone to digital technology[2].

Along with the speed of information delivery until the era of 5 Generation $(5 \mathrm{G}$,) telemedicine was not only used as a media for counseling or broadcasting, telemedicine had evolved into a treatment that was carried out directly between doctors and patients in different places. Telesurgery is a medical method in carrying out long-distance operations relying on $5 \mathrm{G}$ networks that have very little latency combined by robots so that they break through the boundaries of space, especially in the field of surgery.

The first telesurgery was conducted in China, by a surgical expert in Fujian on January 8, 2019. The operation was the appointment of a lab test animal's heart using a robot as an intermediary medium. The expert did it with a distance of about 32 miles. The operation was successful and made new breakthroughs, allowing patients from all corners to make appointments with experts anywhere without fear of lack of health services.

But as we know, not all countries have relied on 5 generation technology, especially in the health sector. Infrastructure problems and expertise in using technology have become a significant obstacle in implementing telesurgery and telemedicine.

In the remaining part of this paper, we will provide an overview of implementing $5 \mathrm{G}$ technology on telemedicine and telesurgery according to other studies as literature, also identify the obstacles that arise in this implementation. we also provide details of the implementation given temporarily and present some evaluation results. And finally we offer conclusions to find solutions for the future.

In Indonesia, the data shows that not a small number of Indonesian patients have to make referrals for operations overseas, such as Singapore, Hong Kong, even China. This is 
due to the lack of health facilities in Indonesia to deal with patients and medical personnel who are experienced in this field. In addition, Indonesia also opens opportunities for foreign medical workers to enter the country of Indonesia. This is certainly a question that we need to pay attention to considering the hope that Indonesia will become an independent and sovereign country, one of which is in the health sector. For this reason, 5G technology with its application in the field of telesurgery will be very helpful in achieving that goal. This paper will discuss how telesurgery technology with $5 \mathrm{G}$ can help improve the quality of patient care facilitation by medical personnel in Indonesia.

\section{LITERATURE REVIEW}

In raising the level of nutrition and public health, it influences the development of medical technology, equipment, theory and disease mitigation [3]. But the scarcity of distribution from inadequate medical resources is a huge obstacle. The challenge that arises is how to overcome the shortage of medical resources in terms of space and time so that an effective distribution of medical resources can be created. Telemedicine is a good option for dealing with existing medical problems. By using audio communications, long distance video and other techniques, it is expected to distribute medical assistance to areas with low medical levels. Thus, areas with inadequate medical knowledge can be helped and improved. In today's modern era, audio and video are not enough, real actions that can be taken to carry out medical activities such as remote operations are needed. Therefore, 5G technology is here to answer that challenge [4]. 5G architecture can be the best way because it can recognize movement in realtime. And can transfer human actions into tactile form without using cables (wireless 5G).

The Network architecture of $5 \mathrm{G}$ is consist of three layers. The first layer is the infrastructure layer, which covered the user terminal such as sensor, smartphone, devices, etc. This layer shows the core network to processing data between platform. The Second layer is the resource cognitive engine. This layer is intended to perform a data analysis trough perception and learning of networks context for user information (resource). Using the resource cognitive layer, system can achieve the high reliability, high flexibility, and scalability of $5 \mathrm{G}$ system, so as to improve user experience and satisfy.

The third layer is the main function layer of the cognitive data engine. The amount of data is an important subject in this layer and we also know as big data. Big data as a source will help cognitive data to reach a certain perception as a deep machine learning material. With capabilities like this, the real world environment and humans can be recognized by the $5 \mathrm{G}$ system by improving the cross data platform. [5].
For M-healts solutions, the role of the $5 \mathrm{G}$ net in designing and developing cellular health solutions (MHealth) is used as machine-to-machine communication (M2). The device or application used is bridged by $5 \mathrm{G}$ to communicate. Key developments in M2M and IoT communication are a focal point for improving health care solutions. M-Health in the near future can produce a significant improvements like surgery with the help of robotsand patient e-monitoring systems [6]. Lee at al, proposed a system where patients need not have to be in the hospital all the time because they can be monitored when at home through the system. In the system, there is an emergency button that can be used by patients in the event of an emergency. The Smart Health Monitoring system also introduces sensors along with emergency buttons that can produce alarms in providing health [7]. 5G technology clearly has the potential to provide platform for better connectivity in field of $\mathrm{M}$-health services. Continuous monitoring of health is need of today's fast pacing world where people do not have time to go hospital and wait for hours. This technology definitely ease some burden from people as well as from hospital. It also reduce health related problems by diagnosing it in early stages. $5 \mathrm{G}$ technology has that kind of infrastructure to handle huge amount of data simultaneously but still improvement always require for any system. Overall, M-Health has huge opportunities in near future.

Telesurgery services in general, are services that categorized as basic services or value-added assistance services. Basic telesurgery services are developed into several parts. (i) absence of haptic feedback: the main console is operated by the surgeon to manipulate the teleoperator using $3 \mathrm{D}$ video technology [9], [10]. (ii) Haptic with indirect feedback: A sensory system that is not intuitive so it requires a deeper learning curve in terms of time. (iii) direct haptic feedback: the system has been automated using a robot equipped with sensors called sensoraptics. With the existence of these sensors, it is expected to improve the performance of telesurgery, especially for beginners because it can help experts in making the right movements and reduce the learning curve [12].

\section{RESEARCH METHODOLOGY}

In this research, researcher willing to breakdown factors that affects customer satisfaction. factors that have been found are expectations, quality of service, promotion and perceived value. The equipment needed to carry out telesurgery consists of several components such as operating robots that are in place of doctors and patients, connecting application between 2 robots in Figure 2, technical expertise in both places, fast internet, and technology support. 


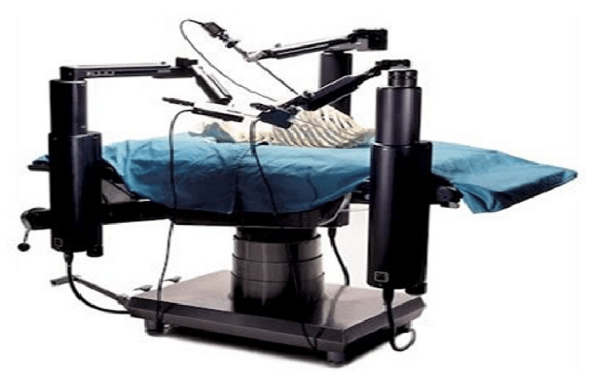

Figure 2 : ZEUS Robot machine

The operating robot used is ZEUS Robot which is placed in the patient's operating room, this robot has 2 cameras that are used as projector to see the real condition on the left and right parts of the robot. The internet speed needed to do this takes $15 \mathrm{Mbps}$, with $4 \mathrm{~g}$ technology there may be a delay, but with $5 \mathrm{~g}$ with latency of only 0.01 second, this is possible. Latency speed test in Table 1.

Table 1 : Latency Objective in 5G-Csys

\begin{tabular}{|l|l|l|l|l|}
\hline & Terminal & $\begin{array}{l}\text { Radio } \\
\text { Interface }\end{array}$ & $\begin{array}{l}\text { Core } \\
\text { Network }\end{array}$ & Total \\
\hline 5C-LM & $0-3 \mathrm{~ms}$ & $2-5 \mathrm{~ms}$ & N/A & $2-10 \mathrm{~ms}$ \\
\hline 5C-MMC & $0-3 \mathrm{~ms}$ & $2-5 \mathrm{~ms}$ & $10-30 \mathrm{~ms}$ & $10-40 \mathrm{~ms}$ \\
\hline 5C-Cloud & $0-3 \mathrm{~ms}$ & $2-5 \mathrm{~ms}$ & $10-40 \mathrm{~ms}$ & $10-50 \mathrm{~ms}$ \\
\hline $\begin{array}{l}\text { 5C- } \\
\text { Cloudlet\&Cloud }\end{array}$ & $0-3 \mathrm{~ms}$ & $5-10 \mathrm{~ms}$ & $10-40 \mathrm{~ms}$ & $20-60 \mathrm{~ms}$ \\
\hline
\end{tabular}

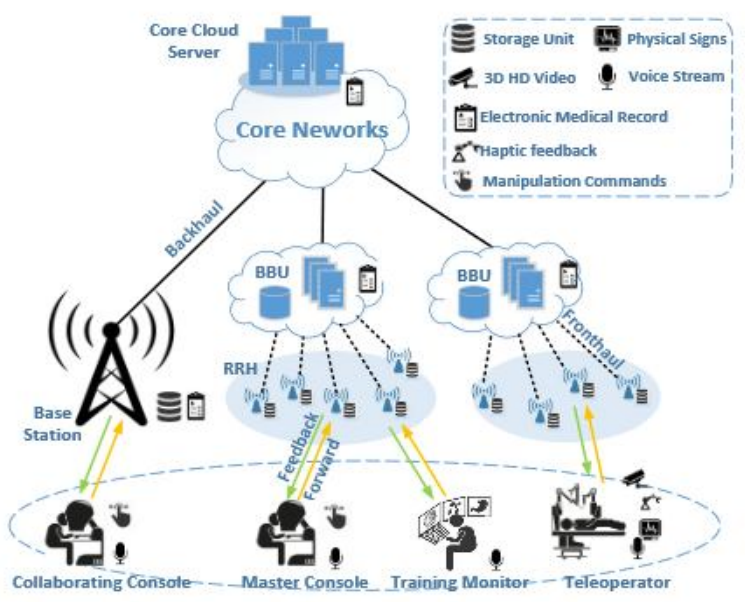

Figure 3 : ZEUS Robot mechanism

This paper will examine the Telesurgery framework developed by Qi Zhang, Jianhui Liu, and Guodong Zhao (2018). In Figure 3 illustrating that a great network architecture that combine the edges with the clouds based on $5 \mathrm{G}$ system communication. D-RAN or C-RAN is used to liaise relations to access the teleoperator and the main console. The da Vinci SI Surgical System provides two consoles that can manipulate teleoperators remotely even geographically separated and this system is used by two surgeons who perform one operation. It can also display demonstration videos for various purposes such as clinical guidance and training.

\section{RESULT AND DISCUSSION}

When applying 5G in telesurgery, there are still many challenges for the implementation. These challenges can include high costs in communication, stable connections, security or privacy and the ability of users to use telesurgery robots.

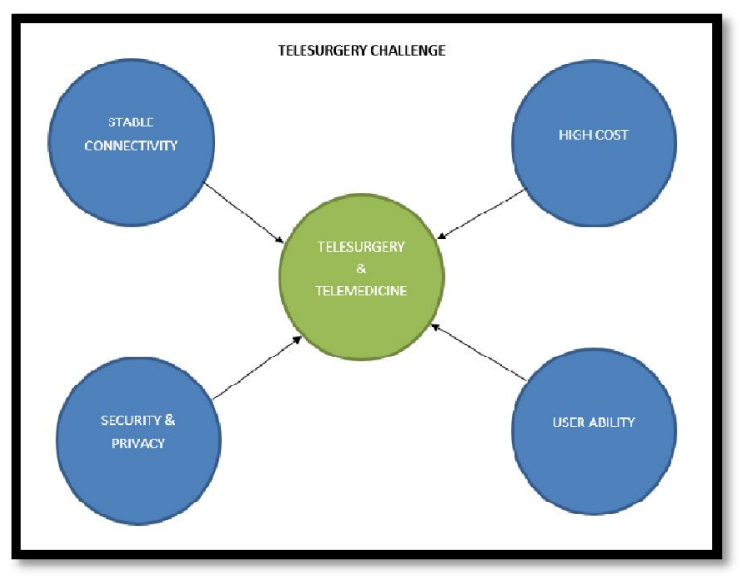

Figure 4 : Telesurgery Challenge

\subsection{High Cost in Communication}

Until now, telesurgery was still not an obligation in medical activities. Expensive costs are one of the main factors. In communication costs specifically networks, it takes \$100,000 - \$200,000 in a year period. This has not been accompanied by other supporting technological equipment such as the provision of robots, computers, and teaching in the application of telesurgery[8].

\subsection{Stable Connections}

The performance of a network is a vital part of telesurgery activity. Especially from latency, packet loss to jitter. As we know that if there are network problems in carrying out telesurgery, it will have fatal consequences[8]. As a standard that the transmission speed with an average bitrate of 1.2 Megabytes per second with a deviation of $95 \mathrm{kbps}$. This experiment is to test if the network is good or medium bad for the system response.

\subsection{Security and Privacy}

The risk of using internet networks in telesurgery is a matter of security and privacy. Connected networks will be very vulnerable in terms of data theft until the system takes over. This is not only detrimental to data theft, but also can endanger patient safety. Therefore, the solution that can be presented is how the system can design cryptography and encrypt security so that the telesurgery can run safely

\subsection{User Ability}

The ability of a surgeon to operate telesurgery equipment is an unavoidable variable. The use of 
robots and conventional surgery is a different matter. It takes skills in the use of computers to the ability to read instruments that are in it.

Changes to the health care paradigm and the expansion of the telemedicine domain will occur with this robotic surgery. Many opportunities in the world of health, especially surgery for this discovery. An advanced robotic telescope system, identifying limiting factors, and summarizing communication are the main discussions of this paper. There are challenges in meeting QoS for multi-modal sensor data and enabling technologies in $5 \mathrm{G}$ communication systems. The important role of $5 \mathrm{G}$ is very clear in overcoming challenges in the world of health.

In developing this telesurgery technology things that need to be considered are not only on technical matters but also on business models that can be developed so that this technology can be commercialized, and more importantly it needs to pay attention to the applicable legal factors and the laws governing the use of this technology. The application of this technology can be started by using dual-polishing, which is an experienced surgeon who can perform complex tasks for remote operations. This scenario can be used because it also has a good business concept and has shorter and cost-effective communication resources.

\section{CONCLUSION}

This article provides an overview of the challenges of implementing telesurgery especially in the use of $5 \mathrm{G}$ networks. From this article, it was stated that there are still a number of things that must be improved so that telesurgery can be maximized by its potential and eliminate the special distance limits in medicine and surgery.

\section{REFERENCES}

[1] R. N. Mitra and D. P. Agrawal, "5G mobile technology: A survey pp. 132-137, 2016. https://doi.org/10.1016/j.icte.2016.01.003

[2] X. Gao, "The Anatomy of Teleneurosurgery in China," vol. 2011, 2011. https://doi.org/10.1155/2011/353405

[3] Alliance, N. 5G White Paper. 2015. Available online: https://www.ngmn.org/uploads/media/NGMN_5G_ White_Paper_V1_0.pdf

[4] Simsek, M.; Aijaz, A.; Dohler, M.; Sachs, J.; Fettweis, G. 5G-enabled tactile internet. IEEE J. Sel. Areas Commun.2016, 34, 460-473.

[5] Chen, M., Yang, J., Hao, Y., Mao, S., \& Hwang, K. (2017). A 5G Cognitive System for Healthcare. Big Data and Cognitive Computing. https://doi.org/10.3390/bdcc1010002
[6] Singh, R., \& Garhewal, R. (2018). Smart M-Health Continuous Monitoring System Using 5G Technology. (December).

[7] J.-V. Lee, Y.-D. Chuah, and K. T. Chieng, "Smart elderly home monitoring system with an android phone,” Int. J. Smart Home, vol. 7, no. 3, pp. 17-32, 2013

[8] Q. Zhang, J. Liu, and G. Zhao, "Towards 5G Enabled Tactile Robotic Telesurgery," no. March, 2018.

[9] J. Marescaux, J. Leroy, F. Rubino, M. Smith, M. Vix, M. Simone, and D. Mutter, "Transcontinental robot-assisted remote telesurgery:feasibility and potential applications",Annals of surgery, vol. 235,no. 4, pp. 487492, 2002.

[10] M. Perez, S. Xu, S. Chauhan, A. Tanaka, K. Simpson, H. Abdul-Muhsin, and R. Smith, "Impact of delay on telesurgical perfor-mance: study on the robotic simulator dvtrainer",International Journal of Computer Assisted Radiology and Surgery, vol. 11, pp. 581587, 2015

[11] A. M. Okamura, "Haptic feedback in robotassisted minimallyinvasive surgery",Current opinion in urology, vol. 19, no. 1, pp.460473, January 2009. https://doi.org/10.1097/MOU.0b013e32831a478c

[12] M. Simsek, A. Aijaz, M. Dohler, J. Sachs, and G. Fettweis, "5G-enabled tactile internet",IEEE Journal on Selected Areas in Communi-cations, vol. 34, no. 3, pp. 460473, March 2016. https://doi.org/10.1109/JSAC.2016.2525398 